\title{
Increased systemic arterial stiffness in patients with chronic thromboembolic pulmonary hypertension
}

\author{
Monika Sznajder, Olga Dzikowska-Diduch, Katarzyna Kurnicka, \\ Marek Roik, Dominik Wretowski, Piotr Pruszczyk, Maciej Kostrubiec \\ Department of Internal Medicine and Cardiology with Venous Thromboembolism Center, \\ Medical University of Warsaw, Poland
}

\begin{abstract}
Background: Chronic thromboembolic pulmonary hypertension (CTEPH) is a complication of venous thromboembolism (VTE) resulting from non-dissolving thromboemboli in the pulmonary arteries. Previous observations indicate a higher prevalence of atherosclerosis and cardiovascular risk factors in patients with VTE and CTEPH. The purpose of the present study was to evaluate the arterial stiffening assessed by pulse wave velocity (PWV), a marker of arterial stiffness, in CTEPH patients in comparison with a matched control group (CG).

Methods: The study group consisted of 26 CTEPH patients ( 9 male and 17 female, age $69 \pm 10$ years) and $22 C G$ (10 male, 12 female, age $67 \pm 8$ years). In all subjects a physical examination, carotid-femoral PWV and transthoracic echocardiography were performed. Right heart catheterization was done in all CTEPH.

Results: Chronic tromboembolic pulmonary hypertension patients had significantly higher $P W V$ than $C G$ $(10.3 \pm 2.5 \mathrm{~m} / \mathrm{s} v \mathrm{~s} .9 \pm 1.3 \mathrm{~m} / \mathrm{s}, p<0.05)$, even though systolic blood pressure was higher in CG $(120 \pm 11 \mathrm{vs}$. $132 \pm 14 \mathrm{mmHg}, p=0.002)$. $P W V$ correlated only with age and pulmonary vascular resistance $(P V R)$ in CTEPH ( $r=0.45, p=0.03$ and $r=0.43, p=0.03$, respectively). Arterial stiffening defined as $P W V>10 \mathrm{~m} / \mathrm{s}$ was found in $11(42 \%) C T E P H$ patients and in 5 (23\%) cases from $C G(p=0.13)$. CTEPH patients with $P W V>10 \mathrm{~m} / \mathrm{s}$ were older $(74 \pm 8$ vs. $66 \pm 10$ years, $p<0.05)$, had decreased oxygen saturation $\left(\mathrm{SaO}_{2} 89\right.$ [73-96]\% vs. 96 [85-98]\%, $\left.p<0.01\right)$ and tended to have higher PVR (8.1 [3.1-14.0] vs. 5.2 [3.1-12.7] HRU, $p=0.10$ ).

Conclusions: Arterial stiffness, assessed with PWV, is increased in CTEPH. The elevated PWV is associated with older age, lower $\mathrm{SaO}_{2}$ and higher PVR in CTEPH. (Cardiol J 2020; 27, 6: 742-748)

Key words: arterial stiffness, pulse wave velocity, chronic thromboembolic pulmonary hypertension, atherosclerosis
\end{abstract}

\section{Introduction}

Chronic thromboembolic pulmonary hypertension (CTEPH) is a rare but severe complication of pulmonary embolism (PE) caused by the persistent obstruction of the pulmonary arteries. CTEPH was observed in $3.8 \%$ of patients within 2 years after acute PE [1].
Recently, the growing number of studies suggested that atherosclerosis and venous thromboembolism (VTE) can have a common etiology involving inflammatory processes [2].

"Classic" risk factors for cardiovascular disease (like smoking, hypertension, diabetes, dyslipidemia, and an unhealthy lifestyle) have an impact on VTE risk [3]. Patients with symptomatic athero-

Address for correspondence: Maciej Kostrubiec, MD, PhD, FESC, Department of Internal Medicine and Cardiology, Medical University of Warsaw, ul. Lindleya 4, 02-005 Warszawa, Poland, tel: +48 22502 1112, e-mail: maciej.kostrubiec@wum.edu.pl 
sclerosis were reported to have an increased risk of VTE, whereas patients with clinical symptoms of VTE more often developed atherosclerosis [2].

The unfavourable structural and functional changes in the peripheral arteries caused by atherosclerosis leads to a reduction in their elastic properties and finally results in their increased stiffness. Aortic stiffening is one of the earliest detectable markers of vascular remodeling [4] and a predictor of cardiovascular events [5]. The predictive value of aortic stiffening measured as carotidfemoral pulse wave velocity (PWV) is considered the gold standard for assessment of the regional arterial stiffening. PWV allows for the prediction of cardiovascular mortality regardless of SCORE stratification $[5,6]$.

Risk factors associated with an increase of arterial stiffness include age [5, 7], hypertension [8] and, to a lesser extent, gender and other classic risk factors of cardiovascular diseases [5]. A recent study has indicated a higher incidence of coronary artery disease in patients with CTEPH when compared to survivors of acute pulmonary embolism with excluded CTEPH [9]. The suggested mechanisms promoting coronary artery disease in patients with persistent, non-resolved organized pulmonary artery thrombi included inflammation, systemic and local hypercoagulability, and endothelial injury. All these factors also play an integral mechanistic role in the pathophysiology of artery stiffening. The aim of the present study was to evaluate systemic arterial stiffening assessed with PWV in CTEPH patients in comparison with a matched control group (CG).

\section{Methods}

Patients with CTEPH diagnosis established in accordance with European Society of Cardiology/ /European Respiratory Society (ESC/ERS) guidelines were included in the study [1]. Completing at least 3 months of effective anticoagulant therapy, a mean pulmonary artery pressure $\geq 25 \mathrm{mmHg}$, and wedge pressure in the pulmonary artery $<15 \mathrm{mmHg}$ in right heart catheterization (RHC) present in patients with pulmonary arterial perfusion defects on imaging examinations confirmed the diagnosis of CTEPH. Patients matched by age, gender, and concomitant diseases associated with atherosclerosis were included in CG. Patients with severe diabetes mellitus, chronic kidney disease, ischemic heart disease, peripheral vascular disease, previous stroke or systemic rheumatic diseases were excluded from the study.
A physical examination, carotid-femoral PWV, transthoracic echocardiography and six-minute walk test were conducted on the same day, while RHC in CTEPH patients performed within $48 \mathrm{~h}$ after PWV. Systemic pulse pressure (PP) was calculated as the difference between systolic blood pressure (SBP) and diastolic blood pressure (DBP).

The study was conducted in accordance with the guidelines of Good Clinical Practice and with the approval of the local Ethics Committee. All patients received detailed information about the study and provided voluntary written consent to participate.

\section{Pulse wave velocity assessment}

Carotid-femoral PWV measurement was performed after a night's rest in a horizontal position with the camera Complior SR (Artech Medical Company, Paris, France) equipped with two TY-306 tonometric sensors recording changes in pressure in the range $0.01-100 \mathrm{~Hz}$, which comprises harmonic frequencies of pressure waves generated at various heart rates. Sensors were placed at the right common carotid artery and right femoral artery [5]. After the detection, registration, and computer processing the impulses were enhanced to draw out a curve of the pulse wave of the carotid artery and the femoral artery. Then, the time delay between the onset of the two pulse waves was automatically assessed and the pulse wave velocity was calculated (the distance between sites of measurements, divided by the delay time). The mean of three distance measurements was included in the analysis.

The value of the PWV indicating $a$ healthy artery is still debatable, however, the ESH/ESC 2013 guidelines proposed $\mathrm{PWV}>10 \mathrm{~m} / \mathrm{s}$ to be considered abnormal [6].

\section{Echocardiography}

Transthoracic echocardiography examination was performed in all patients according to the recommendations of the European Association of Cardiovascular Imaging and the guidelines of the Echocardiography Working Group of Polish Cardiac Society [10, 11]. Phillips iE 33 (Andover, Md., USA) with a 2.5-3.5 MHz transducer was used. The size of both ventricles, ejection fraction (LVEF) and diastolic function of left ventricle, tricuspid annular plane systolic excursion (TAPSE), pulmonary velocity value and pattern, tricuspid regurgitation peak gradient, and the dimensions of the inferior vena cava were assessed. 


\section{Laboratory tests}

Kidney function was estimated according to the results of the glomerular filtration rate (eGFR) calculated from the Modification of Diet in Renal Disease formula from serum creatinine concentration.

\section{Six-minute walk test}

The distance an individual was able to walk over a total of 6 min was measured. Heart rate (HR), blood pressure (BP), oxygen saturation $\left(\mathrm{SaO}_{2}\right)$ and dyspnoea (Borg Dyspnoea Score) were also recorded before and after exertion [1]. Desaturation was defined as the difference between the baseline arterial blood saturation and $\mathrm{SaO}_{2}$ measured immediately after the test.

\section{Right heart catheterization}

An experienced interventional cardiologist performed RHC in patients with CTEPH to confirm the diagnosis. The right atrial, right ventricular, pulmonary artery and wedge pressures were measured. The thermodilution method was used to determine stroke volume (SV), cardiac index (CI), and indexed pulmonary vascular resistance (PVR) [12].

\section{Statistical analysis}

Variables with normal distribution are presented as mean with standard deviation (SD), while variables without such distribution are described as median and range (min-max). The Student t-test or Mann-Whitney test was used for comparisons between the two groups. The Fisher or $\chi^{2}$ tests were used to compare discrete variables, as appropriate. The Spearman or Pearson correlation coefficients were calculated, respectively. A stepforward multivariable analysis was performed to indicate the predictors associated with $\mathrm{PWV}>10 \mathrm{~m} / \mathrm{s}$. All tests were two-sided. Data were considered significant at $\mathrm{p}<0.05$. STATISTICA (StatSoft 13.1, Inc. 2016, Tulsa, OK, USA) software was used for statistical calculations.

\section{Results}

The study group consisted of 26 patients with CTEPH (9 men and 17 women, age $69 \pm 10$ years), while 22 patients ( 10 men, 12 women, age $67 \pm 8$ years) were qualified to CG. Patients with CTEPH presented II-III World Health Organization (WHO) functional class. Dyslipidemia and hypertension were present in $8(30.8 \%)$ and $18(69.2 \%)$ patients with CTEPH and in $10(45.5 \%)$ and $13(59.1 \%)$ of CG, respectively. Total and low-density lipoprotein (LDL) plasma cholesterol concentrations were higher, while high density lipoprotein (HDL) cholesterol was lower in CTEPH patients than in controls. The prevalence of smoking history and diabetes did not differ significantly between groups. The clinical characteristics of studied subjects are presented in Table 1. CTEPH patients presented lower LVEF, decreased TAPSE and increased RV to LV dimensions ratio. Moreover, patients with CTEPH presented lower GFR than CG. The six-minute walk distance was remarkably longer in CG than in CTEPH patients (Table 1). CTEPH patients had significantly higher PWV than controls $(\mathrm{PWV} 10.3 \pm 2.5 \mathrm{~m} / \mathrm{s}$ vs. $9 \pm 1.3 \mathrm{~m} / \mathrm{s}, \mathrm{p}<0.05)$ (Fig. 1), even though SBP and PP were higher in the control group (Table 1).

Pulse wave velocity correlated only with age and PVR in patients with CTEPH $(r=0.45$, $\mathrm{p}=0.03$ and $\mathrm{r}=0.43, \mathrm{p}=0.03$, respectively), while in controls PWV correlated with age and inversely with body mass index $(\mathrm{r}=0.54, \mathrm{p}=0.01$ and $\mathrm{r}=-0.43, \mathrm{p}<0.05$, respectively). Interestingly, there was no correlation between PWV and SBP nor DBP in any group.

There was a borderline correlation between PWV and pulmonary compliance (ratio of SV to $\mathrm{PP})(\mathrm{r}=0.37, \mathrm{p}=0.07)$, while the association between PWV and systemic arterial compliance was nonsignificant $(\mathrm{r}=0.29, \mathrm{p}=0.16)$.

Arterial stiffening defined as PWV $>10 \mathrm{~m} / \mathrm{s}$ was found in 11 (42\%) patients with CTEPH and in $5(23 \%)$ cases from CG. CTEPH patients with $\mathrm{PWV}>10 \mathrm{~m} / \mathrm{s}$ compared to CTEPH patients with $\mathrm{PWV} \leq 10 \mathrm{~m} / \mathrm{s}$ were older $(74 \pm 8$ vs. $66 \pm 10$ years, $\mathrm{p}<0.05)$, had decreased oxygen saturation $\left(\mathrm{SaO}_{2} 89\right.$ [73-96]\% vs. 96 [85-98]\%, p < 0.01) and tended to have higher PVR (8.1 [3.1-14.0] vs. 5.2 [3.1-12.7] HRU, $\mathrm{p} \leq 0.10$ ) (Fig. 2). PWV > $10 \mathrm{~m} / \mathrm{s}$ was not associated with lower $\mathrm{SaO}_{2}$ in controls. Multivariable analysis revealed the only significant predictor of $\mathrm{PWV}>10 \mathrm{~m} / \mathrm{s}$ was $\mathrm{SaO}_{2}$ (odds ratio [OR] 0.90; $95 \%$ confidence interval $[\mathrm{CI}] 0.68-0.95, \mathrm{p}<0.01$ ).

\section{Discussion}

The artery stiffening, associated with increased morbidity and mortality, is an important sign of systemic vascular disease. PWV is an independent predictor of adverse cardiovascular events and allcause mortality. A $1 \mathrm{~m} / \mathrm{s}$ rise of its value increases cardiovascular risk by more than $10 \%$ [13].

The decreased arterial compliance results in an increase of central SBP, limits cardiac output and 
Table 1. Characteristics of the study groups.

\begin{tabular}{|c|c|c|c|}
\hline Parameters & CTEPH $(n=26)$ & $C G(n=22)$ & $\mathbf{P}$ \\
\hline \multicolumn{4}{|l|}{ Clinical characteristics } \\
\hline Gender (male/female) & $9 / 17$ & $10 / 12$ & 0.32 \\
\hline Age [years] & $69 \pm 10$ & $67 \pm 8$ & 0.36 \\
\hline BMI $\left[\mathrm{kg} / \mathrm{m}^{2}\right]$ & $28 \pm 5$ & $29 \pm 4$ & 0.23 \\
\hline Heart rate $[\mathrm{bpm}]$ & $66 \pm 11$ & $68 \pm 12$ & 0.52 \\
\hline Systolic BP [mmHg] & $120 \pm 11$ & $132 \pm 14$ & 0.002 \\
\hline Diastolic BP [mmHg] & $76 \pm 11$ & $79 \pm 8$ & 0.43 \\
\hline Pulse pressure $[\mathrm{mmHg}]$ & $43 \pm 11$ & $53 \pm 12$ & 0.005 \\
\hline eGFR [mL/min] & $62 \pm 13$ & $89 \pm 15$ & 0.0001 \\
\hline $\mathrm{SaO}_{2}[\%]$ & $91 \pm 7$ & $98 \pm 1$ & 0.002 \\
\hline 6MWD [m] & $348 \pm 136$ & $599 \pm 107$ & $<0.0001$ \\
\hline Total cholesterol [mg/dL] & $183 \pm 56$ & $144 \pm 37$ & $<0.01$ \\
\hline LDL cholesterol [mg/dL] & $113 \pm 51$ & $79 \pm 33$ & 0.01 \\
\hline HDL cholesterol [mg/dL] & $55 \pm 15$ & $42 \pm 14$ & $<0.01$ \\
\hline Triglycerides [mg/dL] & $98 \pm 46$ & $128 \pm 57$ & 0.06 \\
\hline Fasting glucose [mg/dL] & $100 \pm 39$ & $96 \pm 12$ & 0.60 \\
\hline History of smoking & $9(35 \%)$ & $3(14 \%)$ & 0.09 \\
\hline Diabetes mellitus & $5(19 \%)$ & $1(5 \%)$ & 0.15 \\
\hline \multicolumn{4}{|c|}{ Echocardiographic parameters } \\
\hline LVEF [\%] & $55 \pm 4$ & $62 \pm 4$ & $<0.0001$ \\
\hline TAPSE $[\mathrm{mm}]$ & $18 \pm 5$ & $24 \pm 3$ & $<0.0001$ \\
\hline TRPG [mmHg] & $74 \pm 22$ & $22 \pm 10$ & $<0.0001$ \\
\hline RV/LV & $1.3 \pm 0.3$ & $0.8 \pm 0.1$ & $<0.0001$ \\
\hline \multicolumn{4}{|c|}{ Right heart catheterization results of CTEPH } \\
\hline $\mathrm{mPAP}[\mathrm{mmHg}]$ & $43 \pm 9$ & & \\
\hline PVR [HRU] & $6.9 \pm 2.9$ & & \\
\hline Cardiac index $\left[\mathrm{L} / \mathrm{min} / \mathrm{m}^{2}\right]$ & $2.7 \pm 0.6$ & & \\
\hline
\end{tabular}

Data are shown as mean \pm standard deviation or number (percentage). $6 \mathrm{MWD}$ - six minute walk distance; BMI — body mass index; BP blood pressure; CG - control group; CTEPH — chronic thromboembolic pulmonary hypertension; eGFR - glomerular filtration rate; HDL - high density lipoprotein; HRU - hybrid reference units (Wood units); LDL — low density lipoprotein; LVEF — left ventricular ejection fraction; mPAP - mean pulmonary artery pressure; PVR - pulmonary vascular resistance; RV/LV - right ventricle/left ventricle; $\mathrm{SaO}_{2}-\mathrm{arterial}$ oxygen saturation; TAPSE — tricuspid annular plane systolic excursion; TRPG — tricuspid regurgitation peak gradient

augments the LV overload. Consequently, coronary blood flow is reduced and cardiac ischemia can develop [4]. The elevation of the PWV in CTEPH patients, compared with the age-matched and adjusted for the presence of comorbidity controls, indicates an increased arterial stiffness in the former. Moreover, the increased arterial stiffness in patients with CTEPH may suggest a greater risk of developing diseases associated with atherosclerosis.

Interestingly, the distinct nature of venous thrombosis and arterial thrombosis is being frequently questioned in recent studies. There is a high number of common risk factors for these diseases [2, 3]. Furthermore, patients with clinical symptoms of VTE, particularly idiopathic, have an increased risk of symptomatic atherosclerosis [2]. Additionally, patients with unprovoked VTE have a higher risk of acute cardiovascular events including acute myocardial infarction and ischemic stroke, than patients with provoked VTE [14].

The value of PWV depends on the thickness of the vascular wall and its elastic properties. It is inversely related to the inner radius of the vessels and blood viscosity. Increased stiffness limits systolic expandability, while a loss of flexibility and cushioning mechanism leads to an increased SBP and a relative reduction of DBP, with a subsequent 


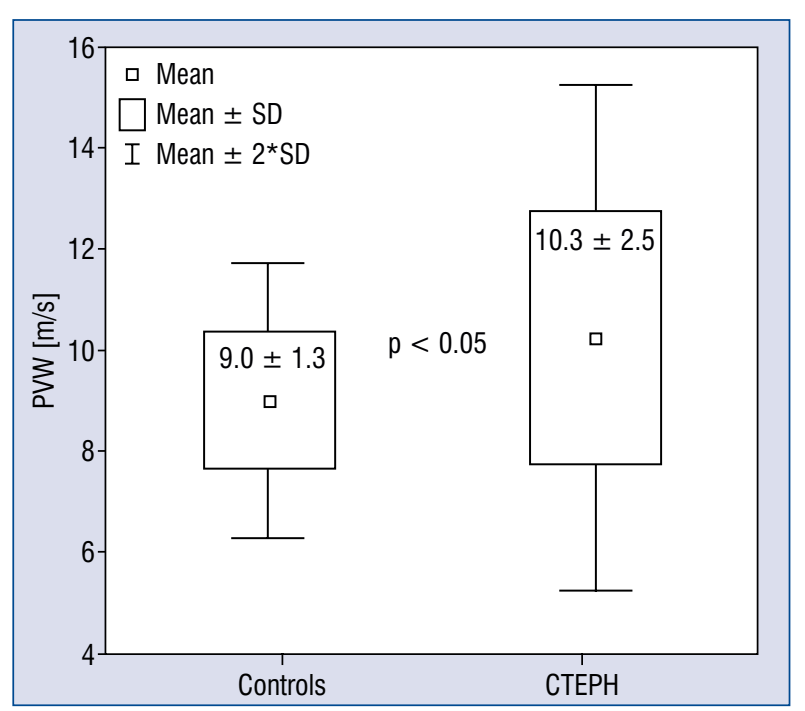

Figure 1. Pulse wave velocity (PWV) value in control group and chronic thromboembolic pulmonary hypertension (CTEPH) patients; SD - standard deviation.

elevation of PP and cardiac afterload [15]. Interestingly, in the current study CTEPH patients were found to have higher PWV despite of lower SBP and PP. Low SBP is common in CTEPH due to low SV.

Vascular stiffening develops from a complex interaction between stable and dynamic changes involving structural and cellular elements of the vessel wall, including the role of scaffolding proteins, extracellular matrix, inflammatory molecules, endothelial cell function, reactive oxidant species and genetics [16].

In CTEPH the pathological lesions are formed by organized thrombi tightly attached to the pulmonary arterial medial layer, replacing the normal intima [17]. The pathophysiology of distal disease may involve predominant obstructions of subseg- mental elastic pulmonary arteries, arteriopathy of small muscular arteries and arterioles distal to non-obstructed vessels and pulmonary arteriopathy of small muscular arteries and arterioles distal to totally or partially obstructed vessels [18]. Distal pulmonary vasculopathy in both the occluded and nonoccluded pulmonary vascular bed is characterized by lesions considered typical for idiopathic pulmonary arterial hypertension. The pathogenesis of these vascular lesions may involve the endothelium, platelet production, release of mediators and altered pulmonary blood flow. Medial hypertrophy, intimal proliferative, and fibrotic changes can be found in CTEPH [19, 20]. However, the down regulation of angiogenic gene expression and the dysfunction of endothelial cells, which may also impair thrombus resolution, were reported as well [21]. Moreover, systemic inflammation seems to be involved in the development of CTEPH [22].

Many patients with CTEPH develop hypoxemic respiratory insufficiency. In the present study PWV correlated with PVR, age and $\mathrm{SaO}_{2}$, however, in multivariable analysis decreased $\mathrm{SaO}_{2}$ was the only significant predictor of PWV $>10 \mathrm{~m} / \mathrm{s}$. The association of vascular stiffening with age was previously well described [5, 7]. The decreased $\mathrm{SaO}_{2}$ can deteriorate endothelium function and increase BP leading to arterial stiffening. Moreover, the low oxygen concentration can cause a rise of PVR and pulmonary arterial pressure [23]. The PVR is related to the geometry of small distal resistive pulmonary arterioles and is considered to reflect the functional status of pulmonary vascular endothelium/smooth muscle cell coupled system. PVR is also positively related to blood viscosity and may be influenced by changes in the perivascular alveolar and pleural pressure [24]. Jujo et al. [20] suggested that a close relationship

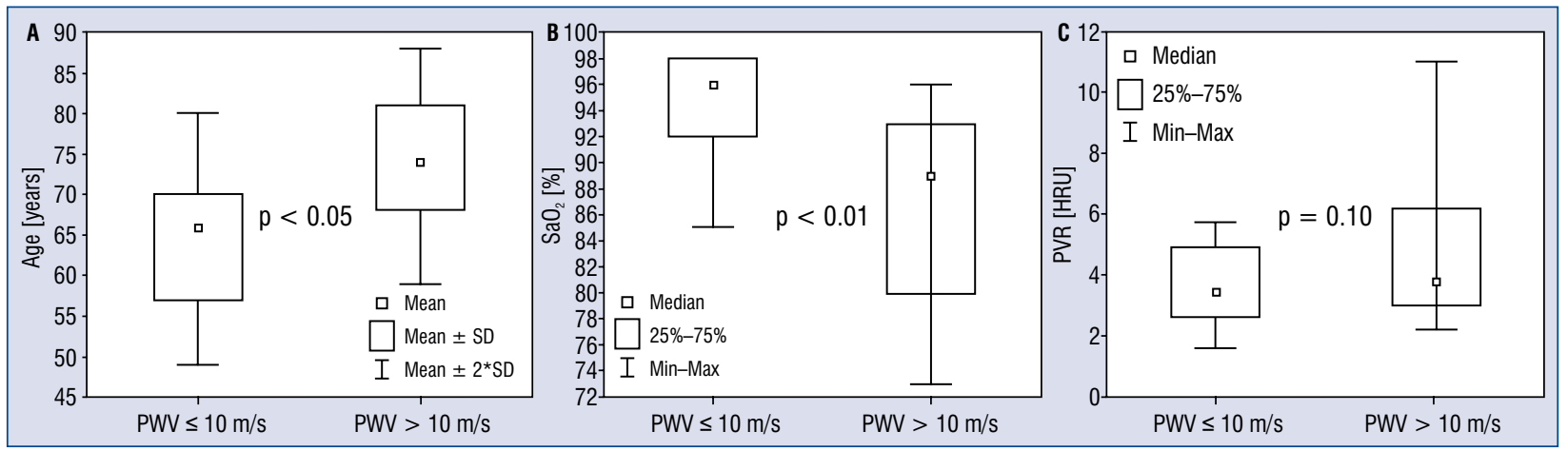

Figure 2. The comparison of age (A), arterial oxygen saturation $\left(\mathrm{SaO}_{2}\right)(\mathbf{B})$, and pulmonary vascular resistance (PVR) (C) between chronic thromboembolic pulmonary hypertension (CTEPH) patients with pulse wave velocity (PWV) above and below/equal to $10 \mathrm{~m} / \mathrm{s}$; SD — standard deviation. 
between severe pulmonary arterial remodeling and elevated PVR could be the result of luminal narrowing of pulmonary muscular arteries. The pathomechanisms underlying CTEPH-related atherosclerotic arterial alternations are not clear. There are no studies available regarding whether thromboembolic pulmonary hypertension triggers mechanisms that may affect systemic arteries such as the aorta. Hou et al. [25] assessed the carotid artery in pulmonary hypertension and revealed that its stiffening might be related to the release of endothelium dependent vasoconstrictors and a decreased concentration of vasodilators. Additionally, in a meta-analysis by Wang et al. [26] which included 3198 patients with chronic obstructive pulmonary disease, markers of subclinical atherosclerosis and cardiovascular risk assessed inter alia with PWV were significantly elevated. The results of the present study point to a significant correlation between arterial stiffening, assessed by PWV, and low $\mathrm{SaO}_{2}$, older age, and increased PVR. They also suggest an association between increased stiffness of the systemic arteries with the occlusion of pulmonary arteries and decreased blood oxygenation in patients with CTEPH.

\section{Limitations of the study}

This is a single center study on a relatively small group of patients with a very rare disease. The observations were not blinded, however, due to a semi-automatic measurement methodology, the influence of the investigator seems negligible. Based on the results of the current study it is impossible to conclude the pathogenesis of arterial stiffening in CTEPH, however, the indicated associations suggest possible mechanisms and directions for further studies.

\section{Conclusions}

Arterial stiffness, a marker of atherosclerosis assessed as pulse wave velocity, is increased in patients with chronic thromboembolic pulmonary hypertension compared to matched controls. The elevated PWV is associated with older age, higher pulmonary vascular resistance and especially with lower oxygen saturation in chronic thromboembolic pulmonary hypertension.

Conflict of interest: None declared

\section{References}

1. Galie N, Humbert M, Vachiery JL, et al. 2015 ESC/ERS Guidelines for the diagnosis and treatment of pulmonary hypertension: The Joint Task Force for the Diagnosis and Treatment of Pulmonary Hypertension of the European Society of Cardiology (ESC) and the European Respiratory Society (ERS): Endorsed by: Association for European Paediatric and Congenital Cardiology (AEPC), International Society for Heart and Lung Transplantation (ISHLT). Eur Heart J. 2016; 37(1): 67-119.

2. Franchini M, Mannucci PM. Association between venous and arterial thrombosis: clinical implications. Eur J Intern Med. 2012; 23(4): 333-337, doi: 10.1016/j.ejim.2012.02.008, indexed in Pubmed: 22560380.

3. Riva N, Donadini MP, Ageno W. Epidemiology and pathophysiology of venous thromboembolism: similarities with atherothrombosis and the role of inflammation. Thromb Haemost. 2015; 113(6): 1176-1183, doi: 10.1160/TH14-06-0563, indexed in Pubmed: 25472800.

4. Cavalcante JL, Lima JAC, Redheuil A, et al. Aortic stiffness: current understanding and future directions. J Am Coll Cardiol. 2011; 57(14): 1511-1522, doi: 10.1016/j.jacc.2010.12.017, indexed in Pubmed: 21453829.

5. Laurent S, Cockcroft J, Van Bortel L, et al. Expert consensus document on arterial stiffness: methodological issues and clinical applications. Eur Heart J. 2006; 27(21): 2588-2605, doi: 10.1093/ eurheartj/ehl254, indexed in Pubmed: 17000623.

6. Mancia G, Fagard R, Narkiewicz K, et al. 2013 ESH/ESC Practice Guidelines for the Management of Arterial Hypertension. Blood Pressure. 2014; 23(1): 3-16, doi: 10.3109/08037051.2014.868629.

7. McEniery CM, Hall IR, Qasem A, et al. ACCT Investigators. Normal vascular aging: differential effects on wave reflection and aortic pulse wave velocity: the Anglo-Cardiff Collaborative Trial (ACCT). J Am Coll Cardiol. 2005; 46(9): 1753-1760, doi: 10.1016/j.jacc.2005.07.037, indexed in Pubmed: 16256881.

8. Simon AC, Levenson J, Bouthier J, et al. Evidence of early degenerative changes in large arteries in human essential hypertension. Hypertension. 1985; 7(5): 675-680, indexed in Pubmed: 4030039.

9. Roik M, Wretowski D, Kostrubiec M, et al. High prevalence of severe coronary artery disease in elderly patients with nonoperable chronic thromboembolic pulmonary hypertension referred for balloon pulmonary angioplasty. Postepy Kardiol Interwencyjnej. 2016; 12(4): 355-359, doi: 10.5114/aic.2016.63637, indexed in Pubmed: 27980550.

10. Lang RM, Badano LP, Mor-Avi V, et al. Recommendations for cardiac chamber quantification by echocardiography in adults: an update from the American Society of Echocardiography and the European Association of Cardiovascular Imaging. J Am Soc Echocardiogr. 2015; 28(1): 1-39.e14, doi: 10.1016/j.echo.2014.10.003, indexed in Pubmed: 25559473.

11. Kasprzak JD, Płońska E, Szyszka A, et al. Echokardiografia w praktyce klinicznej-Standardy Sekcji Echokardiografii Polskiego Towarzystwa Kardiologicznego 2007. Kardiol Pol. 2007; 65(9): 1142-62.

12. Konstantinides SV, Torbicki A, Agnelli G, et al. 2014 ESC guidelines on the diagnosis and management of acute pulmonary em- 
bolism. Eur Heart J. 2014; 35(43): 3033-69, 3069a, doi: 10.1093/ /eurheartj/ehu283, indexed in Pubmed: 25173341.

13. Vlachopoulos C, Aznaouridis K, Stefanadis C. Prediction of cardiovascular events and all-cause mortality with arterial stiffness: a systematic review and meta-analysis. J Am Coll Cardiol. 2010; 55(13): 1318-1327, doi: 10.1016/j.jacc.2009.10.061, indexed in Pubmed: 20338492.

14. Becattini C, Vedovati MC, Ageno W, et al. Incidence of arterial cardiovascular events after venous thromboembolism: a systematic review and a meta-analysis. J Thromb Haemost. 2010; 8(5): 891-897, doi: 10.1111/j.1538-7836.2010.03777.x, indexed in Pubmed: 20095999.

15. Safar M. Tętnice w nadciśnieniu tętniczym. Lippincott-Raven, Philadelphia, 1997.

16. Zieman SJ, Melenovsky V, Kass DA. Mechanisms, pathophysiology, and therapy of arterial stiffness. Arterioscler Thromb Vasc Biol. 2005; 25(5): 932-943, doi: 10.1161/01. ATV.0000160548.78317.29, indexed in Pubmed: 15731494.

17. Fedullo PF, Auger WR, Kerr KM, et al. Chronic thromboembolic pulmonary hypertension. N Engl J Med. 2001; 345(20): 14651472, doi: 10.1056/NEJMra010902, indexed in Pubmed: 11794196.

18. Galiè N, Kim NHS. Pulmonary microvascular disease in chronic thromboembolic pulmonary hypertension. Proc Am Thorac Soc. 2006; 3(7): 571-576, doi: 10.1513/pats.200605-113LR, indexed in Pubmed: 16963536.

19. O'Connell C, Montani D, Savale L, et al. Chronic thromboembolic pulmonary hypertension. Presse Med. 2015; 44(12 Pt 2): e409-e416, doi: 10.1016/.j.lpm.2015.10.010, indexed in Pubmed: 26585271.

20. Jujo T, Sakao S, Ishibashi-Ueda H, et al. Evaluation of the Microcirculation in Chronic Thromboembolic Pulmonary Hyperten- sion Patients: The Impact of Pulmonary Arterial Remodeling on Postoperative and Follow-Up Pulmonary Arterial Pressure and Vascular Resistance. PLoS One. 2015; 10(8): e0133167, doi: 10.1371/journal.pone.0133167, indexed in Pubmed: 26252755.

21. Lang I. Chronic thromboembolic pulmonary hypertension: a distinct disease entity. Eur Respir Rev. 2015; 24(136): 246-252, doi: 10.1183/16000617.00001115, indexed in Pubmed: 26028636.

22. Quarck R, Wynants M, Verbeken E, et al. Contribution of inflammation and impaired angiogenesis to the pathobiology of chronic thromboembolic pulmonary hypertension. Eur Respir J. 2015; 46(2): 431-443, doi: 10.1183/09031936.00009914, indexed in Pubmed: 26113681.

23. Hunter KS, Lammers SR, Shandas R. Pulmonary vascular stiffness: measurement, modeling, and implications in normal and hypertensive pulmonary circulations. Compr Physiol. 2011; 1(3): 1413-1435, doi: 10.1002/cphy.c100005, indexed in Pubmed: 23733649.

24. Chemla D, Castelain V, Hervé P, et al. Haemodynamic evaluation of pulmonary hypertension. Eur Respir J. 2002; 20(5): 1314-1331, indexed in Pubmed: 12449189.

25. Hou Y, Yuan LJ, Xing CY, et al. Carotid arterial stiffness in patients with congenital heart disease-related pulmonary hypertension assesed with rediofrequency data technique. Echocardiography 2015; 32(11): 1676-1680. doi: 10.1111/echo.12925, indexed in Pubmed: 25732062.

26. Wang LY, Zhu YN, Cui JJ, et al. Subclinical atherosclerosis risk markers in patients with chronic obstructive pulmonary disease: A systematic review and meta-analysis. Respir Med. 2017; 123: 18-27, doi: 10.1016/j.rmed.2016.12.004, indexed in Pubmed: 28137492 . 